\title{
NLRP6 function in inflammatory monocytes reduces susceptibility to chemically induced intestinal injury
}

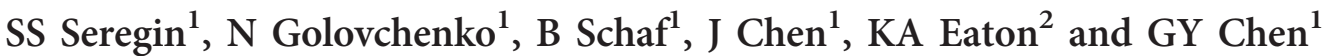

NLRP6 is a member of the Nod-like receptor family, whose members are involved in the recognition of microbes and/or tissue injury. NLRP6 was previously demonstrated to regulate the production of interleukin (IL)-18 and is important for protecting mice against chemically induced intestinal injury and colitis-associated colon cancer. However, the cellular mechanisms by which NLRP6 reduces susceptibility to colonic inflammation remain unclear. Here, we determined that NLRP6 expression is specifically upregulated in Ly6 $\mathrm{C}^{\mathrm{hi}}$ inflammatory monocytes that infiltrate into the colon during dextran sulfate sodium (DSS)-induced inflammation. Adoptive transfer of wild-type (WT) Ly6C ${ }^{\text {hi }}$ inflammatory monocytes into $\mathrm{NIrp6}^{-1-}$ mice was sufficient to protect them from mortality, significantly reducing intestinal permeability and damage. NLRP6-deficient inflammatory monocytes were defective in tumor necrosis factor $\alpha(\mathrm{TNF} \alpha)$ production, which was important for reducing DSS-induced mortality and was dependent on autocrine IL-18 signaling by inflammatory monocytes. Our data reveal a previously unappreciated role for NLRP6 in inflammatory monocytes, which are recruited after DSS-induced intestinal injury to promote barrier function and limit bacteria-driven inflammation. This study highlights the importance of early cytokine responses, particularly NLRP6-dependent and IL-18-dependent TNF $\alpha$ production, in preventing chronic dysregulated inflammation.

\section{INTRODUCTION}

Inflammatory bowel disease (IBD) is a major health problem that results in significant morbidity and mortality due to recurrent episodes of bloody diarrhea, weight loss, and complications from chronic intestinal inflammation, such as cancer. Current approved therapies for IBD rely primarily on symptom management and inhibiting inflammation, but do not address the underlying mechanism behind IBD pathogenesis. The current prevailing model for the pathogenesis of IBD is the development of an imbalance in the microbial community structure within the intestine, or dysbiosis, and/or aberrant immune responses to commensal bacteria in a genetically susceptible host. ${ }^{1} \mathrm{~A}$ better understanding of events that lead to dysregulated inflammation is needed to identify targets for treatment and cure.

Recently, the Nod-like receptor (NLR), NLRP6, has been shown to be important for promoting intestinal homeostasis and for protection against the development of colitis and colitis-associated tumorigenesis in mice. ${ }^{1-4}$ The mechanism by which NLRP6 protects against colitis remains to be fully elucidated. Gene ontogeny analyses have established a link between NLRP6 and intestinal epithelial cell (IEC) repair. ${ }^{4}$ NLRP6 also regulates interleukin (IL)-18 production, which is important for epithelial repair and protection against inflammation-associated colon tumorigenesis. ${ }^{1,2,4-6}$ NLRP6 is highly expressed in IECs, and bone marrow (BM) chimera experiments demonstrated that IL-18 production by epithelial cells is important for reduced susceptibility to dextran sulfate sodium (DSS)-induced intestinal injury and inflammation, suggesting that NLRP6 functions primarily in epithelial cells to promote IL-18 production and epithelial repair. NLRP6 also regulates autophagy within intestinal goblet cells to affect mucus secretion, which contributes to intestinal homeostasis. ${ }^{7}$ However, NLRP6 is also expressed in hematopoietic cell populations, and we have previously demonstrated that NLRP6 functions in BM-derived cells to limit colitis-associated

${ }^{1}$ Division of Hematology/Oncology, Department of Internal Medicine, University of Michigan, Ann Arbor, Michigan, USA and ${ }^{2}$ Department of Microbiology and Immunology, University of Michigan, Ann Arbor, Michigan, USA. Correspondence: GY Chen (gchenry@umich.edu) 
tumorigenesis. ${ }^{1}$ Thus, the specific cell type that is important for NLRP6-mediated protection against the development of colitis and tumorigenesis has not been definitively identified.

In this study, we examined the expression of NLRP6 in various hematopoietic cell populations within the lamina propria (LP) during DSS-induced intestinal injury and determined that inflammatory monocytes, defined as $\mathrm{CD} 11 \mathrm{~b}^{+}$ Ly6 $G^{\text {int }}$ Ly6 $C^{\text {hi }}$ cells, induce NRLP6 expression in response to DSS. More importantly, the adoptive transfer of wild-type (WT) Ly6C hi monocytes into Nlrp6 ${ }^{-1-}$ mice improves survival after DSS treatment. NLRP6-deficient Ly6 $\mathrm{C}^{\text {hi }}$ monocytes had impaired production of tumor necrosis factor $\alpha$ $(\mathrm{TNF} \alpha)$ and reactive oxygen species (ROS), and the injection of recombinant TNF $\alpha(\mathrm{rTNF} \alpha)$ into Nlrp $6^{-/}$mice early during DSS treatment was sufficient for protection against mortality. We further demonstrate that $I l 18^{-/-}$and $I l 18 r 1^{-1-}$ Ly6C $^{\text {hi }}$ inflammatory monocytes have similar defects in TNF $\alpha$ production, and their adoptive transfer into Nlrp6 $6^{-/-}$mice failed to rescue DSS-induced mortality, suggesting that autocrine IL-18 signaling by inflammatory monocytes is important for $\mathrm{TNF} \alpha$ production and protection against acute intestinal injury. Altogether, these studies reveal a protective role for early TNF $\alpha$ production by inflammatory monocytes, which is at least, in part, IL-18- and NRLP6-dependent and is critical for limiting dysregulated commensal-driven intestinal inflammation.

\section{RESULTS}

\section{NLRP6 function in Ly6C ${ }^{\text {hi }}$ inflammatory monocytes} reduces susceptibility to DSS-induced intestinal injury

We previously demonstrated that $N l r p 6^{-1-}$ mice are more susceptible to DSS-induced colitis as well as colitis-associated tumorigenesis after treatment with the carcinogen azoxymethane (AOM) and DSS. ${ }^{1,2,4}$ Furthermore, NLRP6 activity in BM-derived cells was important for limiting inflammationassociated tumors. ${ }^{1}$ To identify the cell type responsible for the protective effects of NLRP6, expression of NLRP6 was measured in different cell populations in the BM and colon LP before and after DSS treatment. We analyzed NLRP6 mRNA expression in IECs, intraepithelial lymphocytes (IELs), $\mathrm{BM}$, and LP cells from WT mice on day 0 and day 10 (at the end of 5 days of $2 \%$ DSS) in the AOM/DSS model of colitisassociated tumorigenesis. ${ }^{1}$ We determined that NLRP6 expression was upregulated in the LP, but not IEC, IEL, or BM cells in response to DSS (Figure 1a). Within the LP, NLRP6 was specifically increased in myeloid cells, and in particular, Ly6 $\mathrm{C}^{\text {hi }}$ monocytes and neutrophils, after DSS treatment with the highest induction in Ly6 $\mathrm{C}^{\text {hi }}$ inflammatory monocytes (Figure 1b). In contrast, NLRP6 expression did not change in $\mathrm{T}$ cells and was undetectable in B cells (Figure 1b). We confirmed the upregulation of NLRP6 expression in LP cells and myeloid cells within the LP in WT mice treated with DSS only, indicating that the observed change in NLRP6 expression is not dependent on AOM (Supplementary Figure $\mathbf{1}$ online).

Upregulation of NLRP6 in response to DSS in Ly6C hi inflammatory monocytes prompted us to investigate if NLRP6 function in this population of cells was important for maintaining intestinal homeostasis. WT Ly6 $\mathrm{C}^{\text {hi }}$ monocytes

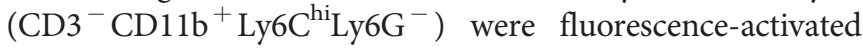
cell sorting (FACS)-sorted to $\sim 99 \%$ purity (Figure 1c), and were adoptively transferred into Nlrp $6^{-/-}$mice on day 3.5 of a 7-day course of high-dose DSS (3.5\%). In contrast to mocktreated Nlrp6 $6^{-/-}$mice, which had a $15 \%$ survival rate after DSS treatment, Nlrp6 ${ }^{-/-}$mice that received Ly6C $\mathrm{C}^{\text {hi }}$ monocytes were protected from lethality with a survival rate of $\sim 70 \%$ (Figure 1c). Consistent with improved survival, $\mathrm{Nlrp6}^{-/-}$mice harboring WT Ly6C ${ }^{\text {hi }}$ monocytes also showed significantly less weight loss (Figure 1d). These results strongly suggest that NLRP6 is upregulated in inflammatory monocytes that are important for reducing DSS-induced mortality. To demonstrate that donor Ly6 $\mathrm{C}^{\text {hi }}$ monocytes can be recruited to the intestine during DSS treatment, we adoptively transferred Ly6C $\mathrm{C}^{\text {hi }}$ monocytes isolated from transgenic mice that express green fluorescent protein (GFP) into Nlrp6 $6^{-/-}$mice during DSS treatment, and detected $\mathrm{GFP}^{+} \mathrm{Ly}_{6 \mathrm{C}}{ }^{\mathrm{hi}}$ monocytes in the LP of recipient mice (Supplementary Figure 2a). As confirmation that the recruitment of inflammatory monocytes into the intestinal LP is important for NLRP6-mediated protection against DSS-induced injury and inflammation, we adoptively transferred $\mathrm{Nlrp6}^{-/-}$mice with Ly6C $\mathrm{C}^{\text {hi }}$ monocytes isolated from mice deficient in CCR2, which is expressed by inflammatory monocytes and is required for their recruitment to sites of inflammation, ${ }^{8}$ and observed no improvement in weights or survival with DSS treatment (Supplementary Figure 2b-d).

Ly6C ${ }^{\text {hi }}$ monocytes can be further characterized by their CX3CR1 expression. During intestinal inflammation, Ly6C ${ }^{\text {hi }} \mathrm{CX} 3 \mathrm{CR} 1^{\text {int }}$ inflammatory monocytes are recruited to the LP. ${ }^{9}$ Consistently after 5 days of DSS, all Ly $6 \mathrm{C}^{\text {hi }}$ monocytes in the LP of WT and Nlrp6 ${ }^{-1-}$ mice express CX3CR1 (Supplementary Figure 3a). To determine CX3CR1 expression in Ly6C $\mathrm{C}^{\text {hi }}$ donor monocytes in adoptive transfer experiments and track their recruitment in the intestine during DSS treatment, we used CX3CR1-GFP mice, in which CX3CR1-expressing cells are labeled with GFP, as donors and $\mathrm{Crr} 2^{-/-}$mice as recipients. Ly6C ${ }^{\text {hi }}$ donor monocytes were mostly CX3CR $1^{\text {int }}(\sim 80 \%)$, (Supplementary Figure 3b), but after transfer into $\mathrm{Ccr} 2^{-/-}$ mice treated with DSS, all donor CCR2 ${ }^{+}$cells in the LP were CX3CR $1^{\text {int }}$ (Supplementary Figure 3c). These results strongly suggest that Ly6 $\mathrm{C}^{\mathrm{hi}}$ monocytes that migrate into the colon in this model and have protective effects in $N l r p 6^{-/-}$mice represent a relatively homogenous population of $\mathrm{CX} 3 \mathrm{CR} 1^{\mathrm{int}}$ inflammatory monocytes.

WT Ly6C ${ }^{\text {hi }}$ monocytes limit tumor growth in NIrp6 ${ }^{-1-}$ mice We had previously reported that Nlrp6 $6^{-/-}$mice developed more colon tumors compared with WT mice in the AOM/DSS model of inflammation-associated tumorigenesis. ${ }^{1}$ To determine whether NLRP6 function in inflammatory monocytes is important for reducing inflammation-associated tumorigenesis, we adoptively transferred WT Ly6 $\mathrm{C}^{\text {hi }}$ monocytes into Nlrp6 $6^{-/-}$mice 3.5 days after the start of each cycle of $2 \%$ DSS 


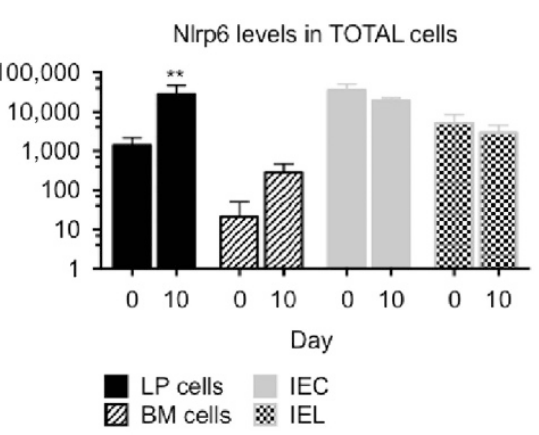

b

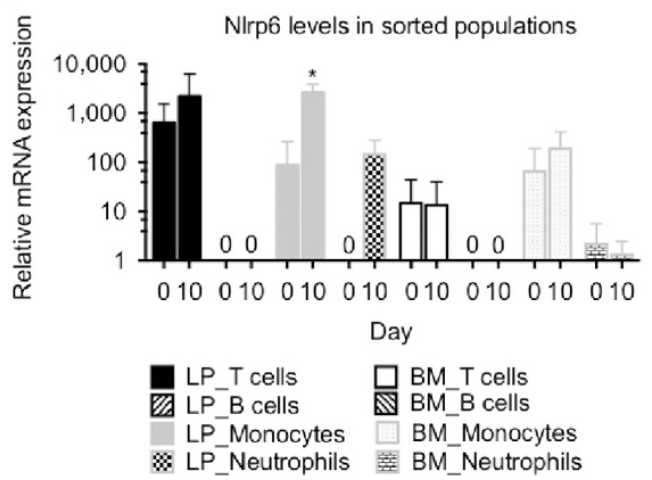

d

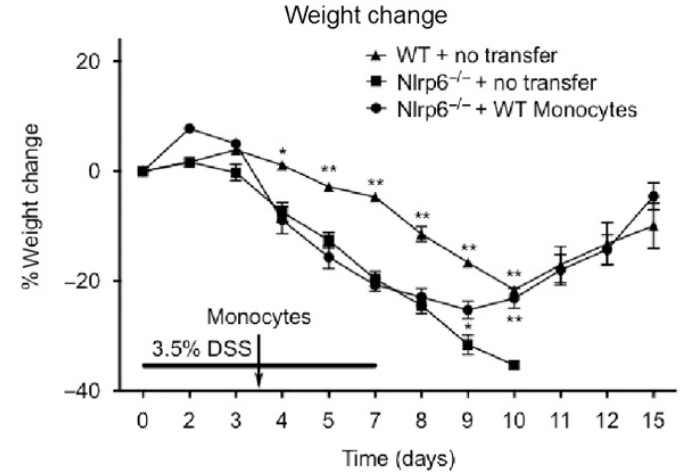

c
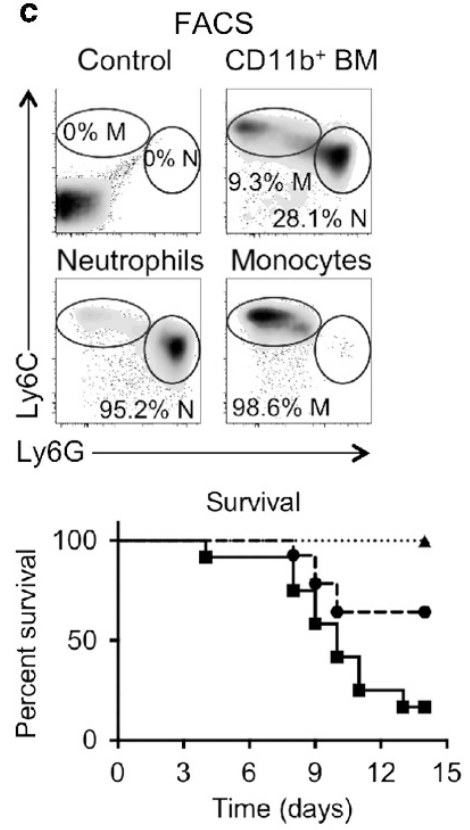

- WT + no transfer

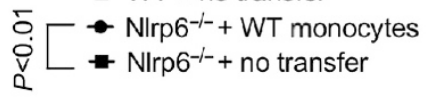

Figure 1 NLRP6 is induced in lamina propria Ly6C ${ }^{\text {hi }}$ monocytes during dextran sulfate sodium (DSS)-induced inflammation, and is important for reducing susceptibility to colitis. (a) NLRP6 levels were measured in bone marrow (BM), lamina propria (LP), intestinal epithelial cells, and intraepithelial lymphocytes of wild-type (WT) mice at day 0 and day 10 of azoxymethane (AOM)/DSS by qPCR. (b) NLRP6 expression was measured in

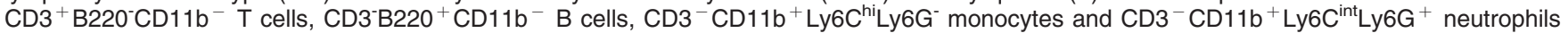
within the BM and LP. Data are representative of three independent experiments; $n=11$ for day $0, n=10$ for day 10 . ${ }^{\star} P<0.05$, ${ }^{* *} P<0.001$, respectively, as compared with day 0 time point of the same genotype. (c) Representative plots of Ly6C vs. Ly6G staining of CD11b ${ }^{+}$BM cells (top). Kaplan-Meier survival curves of mice treated with 7 days of $3.5 \%$ DSS (bottom). (d) Percent weight change with $3.5 \%$ DSS administration. Data are representative of two independent experiments; $n=15, n=24, n=14$ for WT, Nlrp6 ${ }^{-/-}$and NIrp6 ${ }^{-1-}+$ WT Ly6C ${ }^{\text {hi }}$ monocytes groups respectively. ${ }^{\star} P<0.05$,

${ }^{\star \star} P<0.001$, respectively, as compared with NIrp6 ${ }^{-1-}$.

(Figure 2a). As previously published, Nlrp6 ${ }^{-1-}$ mice developed more tumors compared with WT mice (Figure $2 \mathbf{b}){ }^{1}$ Adoptive transfer of WT Ly6 $\mathrm{C}^{\text {hi }}$ monocytes resulted in fewer tumors; however, this did not reach statistical significance (Figure 2b). The transfer of WT Ly6C ${ }^{\text {hi }}$ monocytes into Nlrp6 $6^{-/-}$mice did result in a significant reduction in tumor sizes with fewer tumors greater than $2 \mathrm{~mm}$ in size compared with that in $\mathrm{Nlrp6}^{-1-}$ mice (Figure 2c), and reduced weight loss early during AOM/DSS treatment (days 14-26, Figure 2d).

\section{NLRP6 function in Ly6 $\mathrm{C}^{\text {hi }}$ monocytes promotes resistance against DSS-induced damage.}

To determine the effects of WT Ly6 $\mathrm{C}^{\text {hi }}$ monocytes within the colon of $\mathrm{Nlrp6}^{-1-}$ mice during the acute inflammatory response to DSS, we looked specifically on day 10 of the AOM/ DSS model of tumorigenesis when mice have completed 5 days of $2 \%$ DSS before the onset of chronic, dysregulated inflammation in Nlrp6 ${ }^{-/-}$mice. ${ }^{1}$ Increased intestinal permeability is a classical hallmark of DSS-induced IEC damage. ${ }^{1,2,4}$ To determine if the adoptive transfer of WT Ly6 $\mathrm{C}^{\text {hi }}$ monocytes 
a

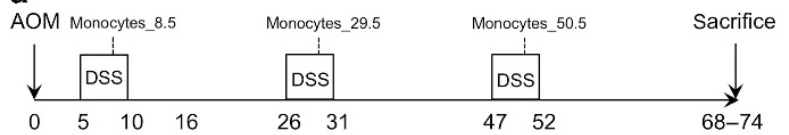

b
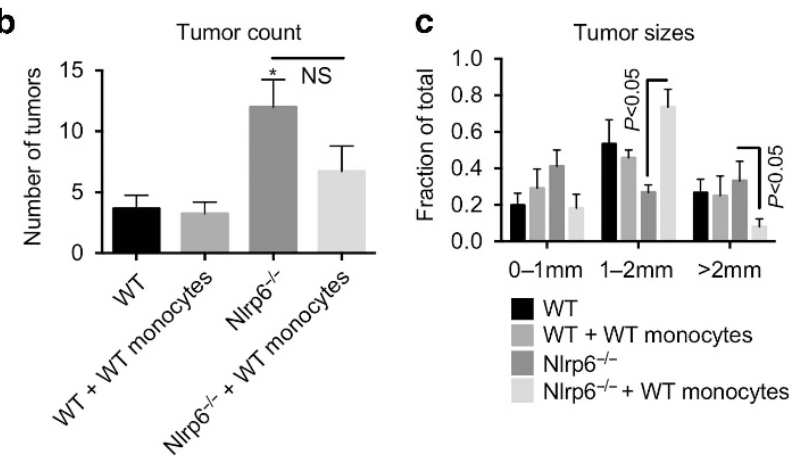

d

d $40 \quad$ Weight change

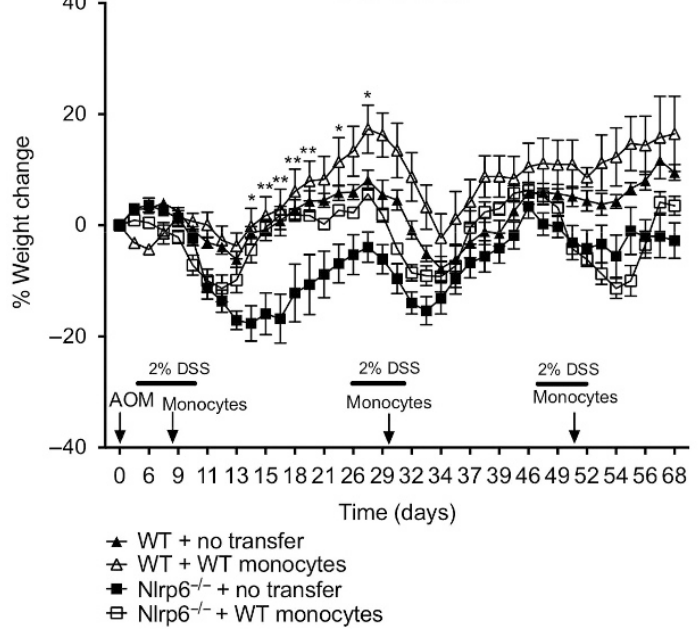

Figure 2 Adoptive transfer of wild-type (WT) Ly6 $\mathrm{C}^{\text {hi }}$ monocytes into Nlrp6 ${ }^{-/-}$mice significantly reduces tumor growth in azoxymethane (AOM)/DSS (dextran sulfate sodium) model of colitis-associated tumorigenesis. (a) Age- and sex-matched NIrp6 ${ }^{-1-}$ and WT mice were treated with AOM followed by three 5 -day cycles of $2 \%$ DSS. Sorted Ly6C ${ }^{\text {hi }}$ monocytes were injected i.v. 3.5 days after the start of each DSS cycle. Three weeks after the last cycle of DSS mice were sacrificed and tumors grossly counted with a stereomicroscope. Number (b) and size (c) of tumors are shown. (d) Percent weight change during AOM/DSS treatment. Data are representative of three independent experiments, $n=15, n=10$, $n=19, n=22$ for WT, WT + WT Ly6C ${ }^{\text {hi }}$ monocytes, NIrp6 ${ }^{-1-}$ and NIrp6 ${ }^{-/-}+$WT Ly6C ${ }^{\text {hi }}$ monocytes groups respectively. ${ }^{*} P<0.05$, ${ }^{* \star} P<0.001$, respectively, as compared with WT (b) or NIrp6 ${ }^{-1-}$ mice (d).

affected intestinal permeability, mice were gavaged with FITCdextran and its passage into the peripheral circulation was measured. As demonstrated previously, ${ }^{1}$ naive WT and Nlrp6 $6^{-/-}$mice had very low levels of serum fluorescence indicative of an intact epithelial barrier, whereas DSS-treated mice, especially Nlrp $6^{-/-}$mice, had higher levels of serum fluorescence suggesting increased intestinal permeability (Figure 3a). ${ }^{1}$ Importantly, the intestinal permeability of Nlrp6 $6^{-1-}$ mice adoptively transferred with WT Ly6C ${ }^{\text {hi }}$ monocytes was similar to that of WT mice after exposure to DSS, and was significantly reduced compared with that of mock-treated Nlrp6 $6^{-/-}$mice (Figure 3a). Consistently, there was a reduction in bacterial translocation into the mesenteric lymph nodes (MLNs) as measured by qPCR in Nlrp6 $6^{-/-}$mice adoptively transferred with WT Ly6C $\mathrm{Ci}^{\mathrm{i}}$ monocytes (Figure 3b). Levels of fecal lipocalin-2, a surrogate marker of intestinal epithelial damage, ${ }^{10}$ were also reduced in $N l r p 6^{-/-}$ mice adoptively transferred with WT Ly6C $\mathrm{C}^{\text {hi }}$ monocytes (Figure 3c). Histological scoring of colitis demonstrated reduced intestinal epithelial damage and inflammation in the presence WT Ly6C ${ }^{\text {hi }}$ monocytes (Figure 3d). Thus, NLRP6 activity in Ly6C ${ }^{\text {hi }}$ inflammatory monocytes is important in limiting DSS-induced damage.

\section{NIrp6 $^{-/-}$Ly6C $^{\text {hi }}$ inflammatory monocytes have impaired TNF $\alpha$ and ROS production}

Inflammatory Ly6C $\mathrm{C}^{\text {hi }}$ monocytes perform critical functions during inflammation, including phagocytosis, production of ROS, and secretion of cytokines. ${ }^{11}$ These functions limit bacterial translocation, promote tissue repair, and ultimately, help to resolve inflammation. To determine whether $N \operatorname{lrp} 6^{-1-}$ Ly6C $\mathrm{C}^{\text {hi }}$ inflammatory monocytes have a defect in phagocytosis, Ly6C ${ }^{\text {hi }}$ monocytes were isolated from naive and AOM/DSStreated WT and Nlrp6 ${ }^{-/-}$mice (day 10). ${ }^{1}$ In agreement with previous studies, we observed no defect in phagocytosis as BMsorted Ly6C ${ }^{\text {hi }}$ monocytes from WT and Nlrp6 ${ }^{-/}$mice exhibited equivalent levels of internalized $\mathrm{GFP}^{+}$Escherichia coli (Figure 4a). ${ }^{12}$ However, ROS production was significantly reduced in BM-derived and LP-isolated Nlrp6 ${ }^{-/-}$Ly6C $^{\text {hi }}$ monocytes as compared with WT Ly6C ${ }^{\text {hi }}$ monocytes after DSS treatment (Figure $\mathbf{4 b}, \mathbf{c}$ ). We next measured levels of various cytokines produced by LP inflammatory Ly6C ${ }^{\text {hi }}$ monocytes isolated from AOM/DSS-treated Nlrp6 ${ }^{-/}$mice and cultured overnight. Nlrp6 $6^{-/-}$LP-derived inflammatory monocytes exhibited decreased early production of $\mathrm{TNF} \alpha$ at the completion of DSS, whereas other cytokines such as IL-6 and IL-1 $\beta$ were produced at levels similar to that by WT Ly6C ${ }^{\text {hi }}$ monocytes (Figure 4d). Consistently, TNF $\alpha$ mRNA expression was also significantly reduced in $N l r p 6^{-/-}$LP Ly6C $^{\text {hi }}$ monocytes (Figure 4e). By day 16, however, approximately 1 week after completion of DSS, TNF $\alpha$ levels were no longer significantly different between WT and Nlrp $6^{-/-}$inflammatory monocytes, suggesting that NLRP6-independent pathways may be compensating (Figure 4d). The defect in TNF $\alpha$ production was specific to Nlrp $6^{-/-}$inflammatory monocytes as $N l r p 6^{-/-}$

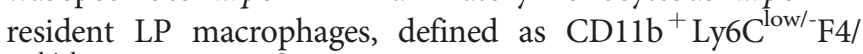
$80^{\text {high }}$ Ly6G $^{-}$cells, ${ }^{9}$ exhibited no differences in cytokine production compared with WT (Supplementary Figure 4). In mice treated with 5 days of DSS alone, we observed similar defects in TNF $\alpha$ production, but not IL-1 $\beta$, by Nlrp6 ${ }^{-/-}{\text {Ly } 6 C^{\text {hi }}}^{-}$ monocytes associated with increased fecal lipocalin levels in Nlrp $6^{-/-}$mice (Supplementary Figure 5).

To determine if NLRP6 upregulation is associated with TNF $\alpha$ production, we sorted Ly6 $\mathrm{C}^{\mathrm{hi}}$ monocytes from the BM of WT mice, and stimulated them with rosiglitazone, which was previously demonstrated to increase NLRP6 expression in colon epithelial cells. ${ }^{13}$ We indeed observed upregulation of NLRP6 

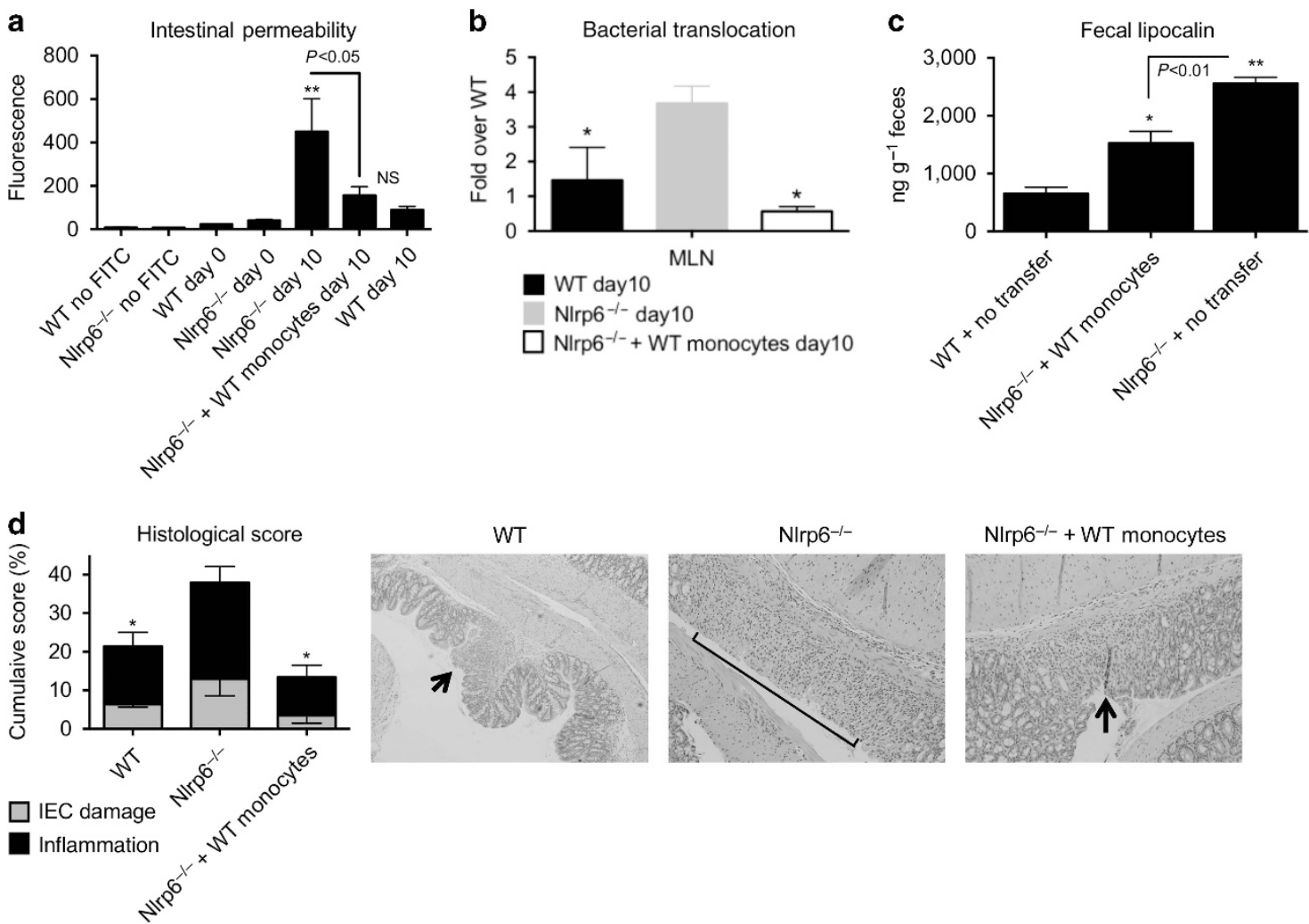

WT
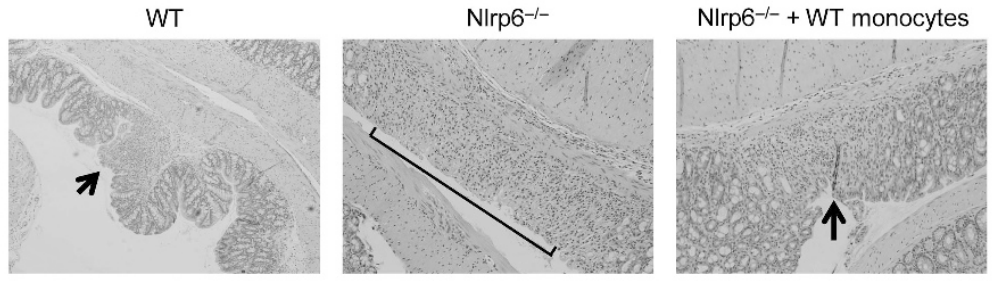

Figure 3 Adoptive transfer of wild-type (WT) Ly6 $\mathrm{C}^{\text {hi }}$ monocytes into Nlrp6 ${ }^{-/-}$mice limits bacterial translocation and intestinal damage. Age- and sexmatched N/rp6 $6^{-1-}$ and WT mice were treated with azoxymethane (AOM) followed by 5 days of $2 \%$ dextran sulfate sodium (DSS). WT Ly6C ${ }^{\text {hi }}$ monocytes were adoptively transferred into NIrp6 ${ }^{-1-}$ mice 3.5 days after start of DSS. (a) Mice were gavaged FITC-dextran at end of 5 days of DSS followed by serum collection and measurement of fluorescence $4 \mathrm{~h}$ later. (b) Normalized levels of total bacteria/mesenteric lymph node as measured by quantitative PCR after 5 days of $2 \%$ DSS. (c) Fecal lipocalin-2 levels as measured by ELISA (enzyme-linked immunosorbent assay) after 5 days of DSS. (d) Histological inflammatory scores based on extent of inflammatory cell infiltration and intestinal epithelial cell damage; Micrographs of H\&E sections are at $200 \times$ magnification. Arrow points to focal erosion with inflammatory infiltrate. Bracket indicates ulcerated epithelium with inflammatory infiltrate in lamina propria and submucosa. Data are representative of three independent experiments, $n=15, n=16, n=20$ for WT, NIrp6 ${ }^{-1}$ - and NIrp $6^{-1-}+$ WT Ly6 $\mathrm{C}^{\text {hi }}$ monocytes groups respectively. ${ }^{\star} P<0.05$, ${ }^{\star \star} P<0.001$, respectively, as compared with day 0 of both genotypes (a), or as compared with NIrp6 ${ }^{-/-}$(b,c) or as compared with WT (d).

expression when WT monocytes were treated with rosiglitazone (Supplementary Figure 6a) along with increased TNF $\alpha$ production in WT macrophages, which did not occur to the same extent in Nlrp6 ${ }^{-1-}$ Ly6C $^{\text {hi }}$ monocytes (Supplementary Figure $6 \mathbf{b}$ ), suggesting that the TNF $\alpha$ response is indeed partially NLRP6 dependent. We next determined whether a defect in the recruitment of Nlrp6 $6^{-1-}$ inflammatory monocytes into the LP was associated with decreased TNF $\alpha$ production. However, examination of monocyte populations in the LP of WT and Nlrp6 $6^{-/-}$mice on day 0 and day 10 after completion of 5 days of DSS showed, in fact, increased numbers of Ly6C ${ }^{\text {hi }}$ monocytes recruited to the LP of $N l r p 6^{-/-}$mice (Supplementary Figure 7). To determine if the impairment in TNF $\alpha$ production by Nlrp6 $6^{-/-}$Ly6C $^{\text {hi }}$ monocytes was not a result of global deficiency in cytokine responses, we isolated inflammatory Ly6 $\mathrm{C}^{\text {hi }}$ monocytes from the LP of WT and Nlrp6 $6^{-1-}$ mice on day 10 of AOM/DSS treatment and performed gene expression analysis by microarray. Functional pathway analysis revealed increased mRNA expression of several cytokines and chemokines in Nlrp6 $6^{-/-}{\text {Ly } 6 C^{\text {hi }}}$ cells, while TNF $\alpha$ was one of the few cytokines that was the most reduced (Supplementary Figure 8).

\section{Early production of TNF $\alpha$ is important for resistance against DSS-induced intestinal injury}

Elevated levels of TNF $\alpha$ have frequently been associated with disease severity in chronic IBD patients; ${ }^{14}$ however, TNF $\alpha$ also promotes epithelial restitution after DSS-induced injury. ${ }^{15}$ To determine whether the early production of $\mathrm{TNF} \alpha$ by inflammatory monocytes is important for protection against intestinal damage and DSS-induced mortality, Nlrp6 $6^{-1-}$ mice were injected with $\mathrm{rTNF} \alpha$ on days 3-7 during a 7-day course of high dose 3.5\% DSS. As expected, the administration of rTNF $\alpha$ into WT mice had no effect on their survival (Figures 5a,b). In contrast, Nlrp6 $6^{-/-}$mice that received rTNF $\alpha$ showed significant improvement in survival with decreased weight loss compared with $N l r p 6^{-1-}$ mice that did not receive supplemental $r T N F \alpha$. There was reduced colonic inflammation and damage as suggested by decreased lipocalin-2 levels as well as less bacterial translocation into the MLNs in Nlrp6 $6^{-/-}$mice that received $r \mathrm{TNF} \alpha$ (Figure 5c,d). Finally, histologic examination of colons revealed a significant improvement in inflammatory scores for Nlrp6 $6^{-/-}$mice that received $r \mathrm{TNF} \alpha$ after 5 days of $2 \%$ DSS (day $10 \mathrm{AOM} / \mathrm{DSS}$ model), reflecting reduced levels of epithelial damage, hyperplasia, and inflammation (Figure 5e). Altogether, 
a

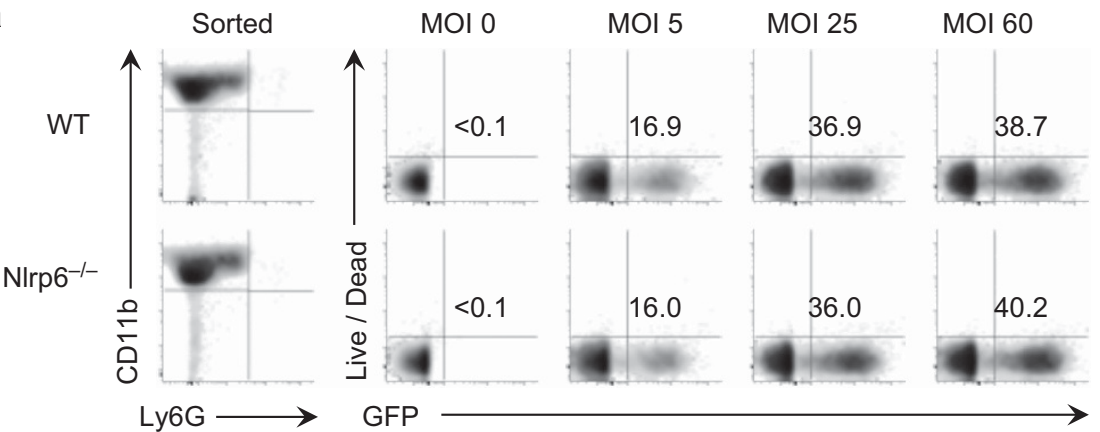

b
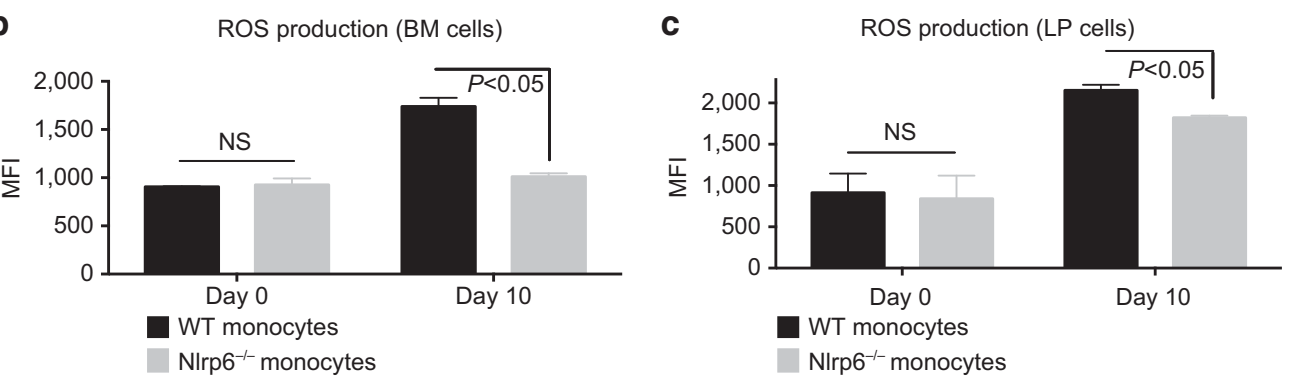

d

TNF $\alpha$

IL6

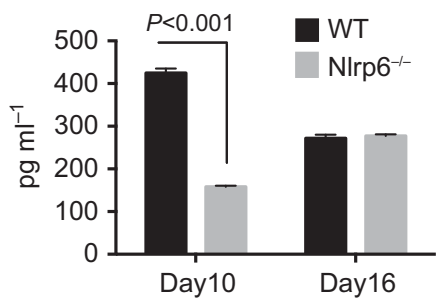

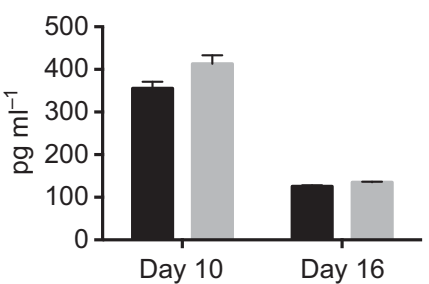

IL1 $\beta$

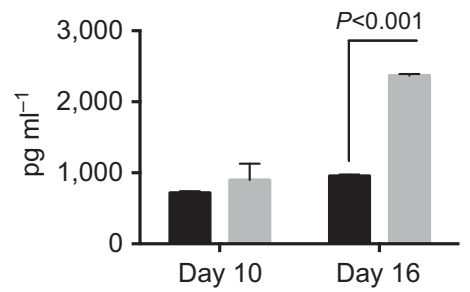

e

LP Ly6C ${ }^{\text {hi }}$ monocytes (Day 10)

LP Ly6C ${ }^{\text {hi }}$ monocytes (Day 16)
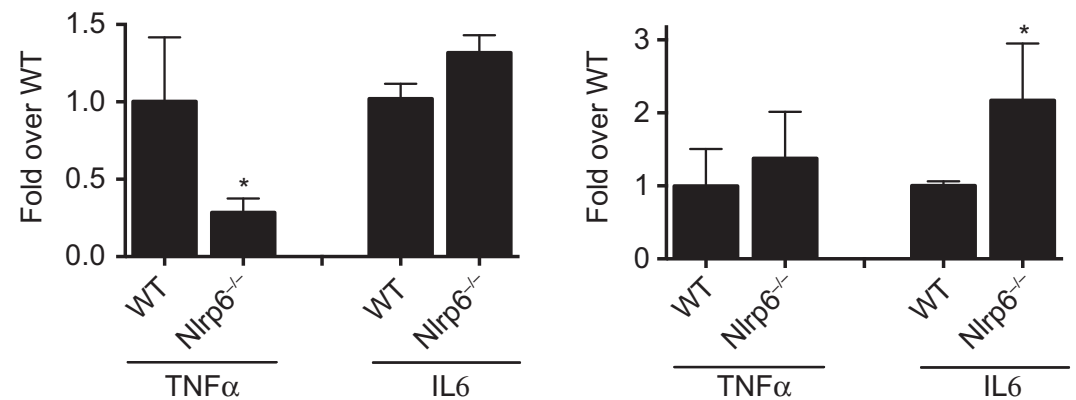

Figure 4 NLRP6-deficient Ly6C ${ }^{\text {hi }}$ monocytes have reduced ROS and TNF $\alpha$ production during the acute inflammatory response to dextran sulfate

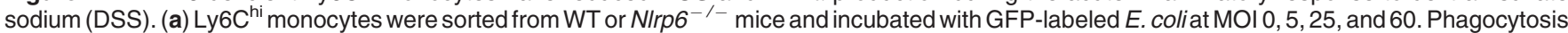

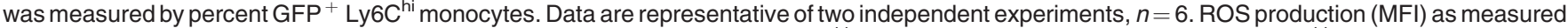
by CellROX deep red in bone marrow- (b) or lamina propria (LP)-derived (c) Ly6C ${ }^{\text {hi }}$ monocytes at the indicated time points. Ly6C ${ }^{\text {hi }}$ monocytes from WT or NIrp6 ${ }^{-1-}$ mice were sorted from the LP after 5 days of $2 \%$ DSS (day 10, AOM/DSS model) or 6 days after completion of DSS (day 16, AOM/DSS model) and cytokine levels were measured by ELISA (enzyme-linked immunosorbent assay) (d) and by qPCR (e). Data are representative of at least three independent experiments, mean \pm s.e.m.; $n=14 /$ genotype, (day 10); $n=12 /$ genotype (day 0 ); $n=8 /$ genotype (day 16 ). * $P<0.05$, as compared with WT.

these results suggest that NLRP6-dependent production of TNF $\alpha$ by WT inflammatory monocytes is important for limiting intestinal damage and bacterial translocation, thereby reducing DSS-induced inflammation and mortality.

\section{Production of TNF $\alpha$ by Ly6 $\mathrm{C}^{\text {hi }}$ inflammatory monocytes is dependent on IL-18 signaling}

We have previously demonstrated that $N l r p 6^{-/-}$mice have impaired production of mature IL-18, which is important for epithelial repair to limit commensal-driven inflammation. ${ }^{1}$ Although it was previously shown that IL-18 is predominantly produced by the intestinal epithelium, ${ }^{3} \mathrm{LP}$ cells are also capable of secreting IL-18. ${ }^{16}$ Importantly, we also observed reduced levels of IL-18 production by Ly6 ${ }^{\text {hi }}$ inflammatory monocytes isolated from DSS-treated Nlrp6 ${ }^{-/-}$mice compared with that from WT (Supplementary Figure 9). To determine if IL-18 production by Ly $6 \mathrm{C}^{\text {hi }}$ monocytes is important for resistance 
a

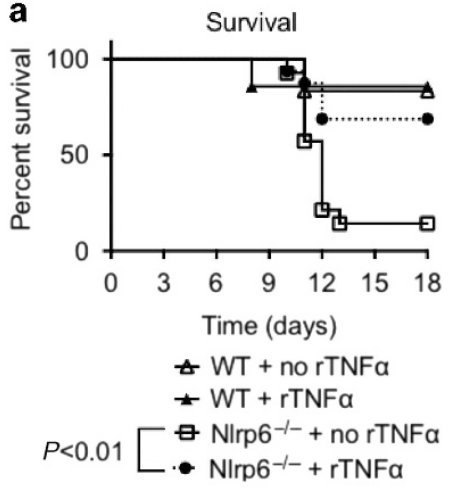

C Fecal lipocalin

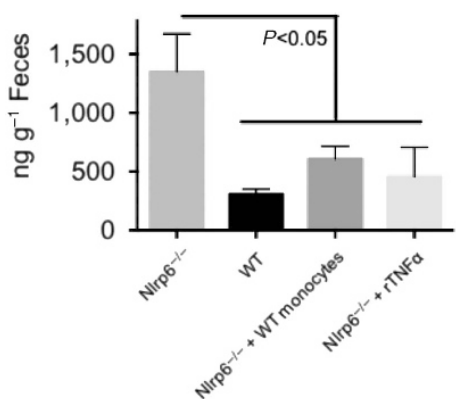

d Bacterial translocation (MLN)

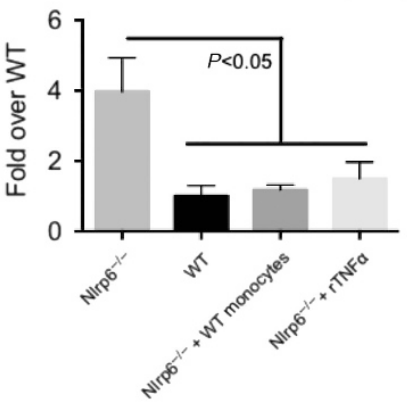

b

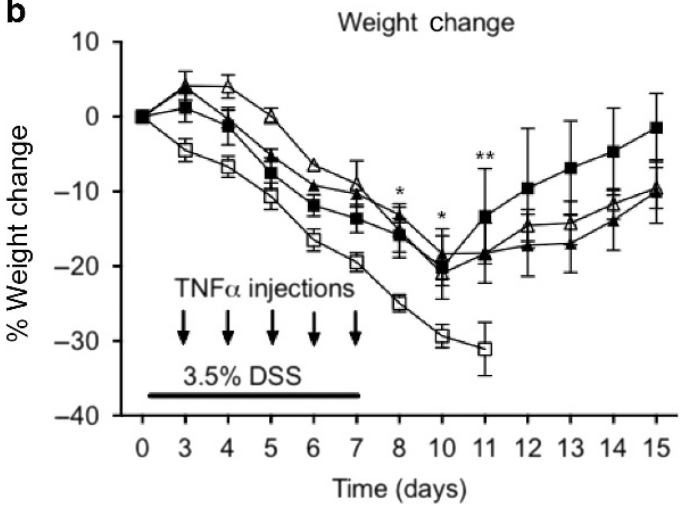

Ð NIrp6 $6^{-1-}+$ no rTNFa

- Nlrp6 $6^{-1}+$ rTNFa

$\triangle W T+$ no rTNFa

$\leftarrow W T+r T N F a$

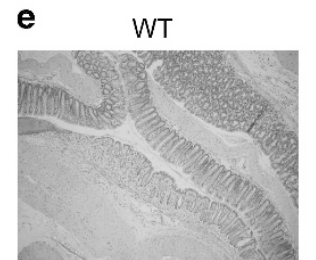

Nirp6-I- + WT monocytes

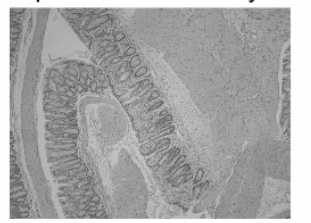

Histological score

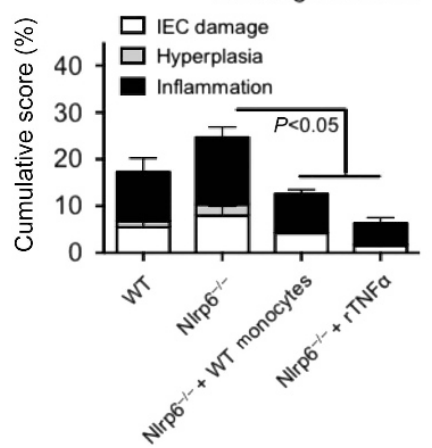

Figure 5 Recombinant TNF $\alpha$ administration into Nlrp6 ${ }^{-/-}$mice early during dextran sulfate sodium (DSS) treatment reduces susceptibility to DSSinduced colitis. (a) Kaplan-Meier survival curves and (b) percent weight change of age- and sex-matched WT and NIrp6 ${ }^{-/-}$mice treated with $3.5 \%$ DSS for 7 days with or without rTNF $\alpha$ injections given on days 3-7. Data are representative of two independent experiments, mean \pm s.e.m.; $n=15, n=16$ for NIrp6 $6^{-\prime-}$ and NIrp6 $6^{-\prime-}+1 \mu \mathrm{g} /$ mouse rTNF $\alpha$ respectively; $n=5$ for WT and WT $+1 \mu \mathrm{g}$ per mouse rTNF $\alpha$ (c) Fecal lipocalin-2 levels measured in WT and NIrp6 ${ }^{-1-}$ mice (day 10, azoxymethane (AOM)/DSS model) after adoptive transfer of Ly6C ${ }^{\text {hi }}$ monocytes or $1 \mu \mathrm{g}$ TNF given on days 3-5 of DSS (d) Normalized levels of total bacteria/mesenteric lymph node as measured by qPCR after 5 days of $2 \%$ DSS (day 10 AOM/DSS model). (e) H\&E micrographs at $100 \times$ after 5 days of $2 \%$ DSS (top) with histological scoring are shown. Bracket indicates diffuse ulceration with inflammatory infiltrate extending throughout the lamina propria and submucosa; $n=5, n=6, n=6, n=7$ for WT, NIrp6 ${ }^{-/-}$, Nlrp6 ${ }^{-1-}+1 \mu$ g per mouse rTNF $\alpha$, and Nlrp6 ${ }^{-/-}+$WT Ly6C $^{\text {hi }}$ monocytes groups respectively.

to DSS-induced mortality, Nlrp6 $6^{-1-}$ mice were adoptively transferred with $I l 18^{-/-}$Ly6C $\mathrm{Ci}^{\text {hi }}$ monocytes, and survival of these mice was compared with that of mock-transferred Nlrp6 $6^{-1-}$ and WT mice after 7 days of 3.5\% DSS (Figure 6a). Nlrp6 ${ }^{-1-}$ mice that received $I l 18^{-/-}$monocytes had similar levels of mortality and weight loss compared with $N \operatorname{lrp} 6^{-/-}$ mice (Figures 6a and b). Interestingly, Ly6 $\mathrm{C}^{\text {hi }}$ monocytes isolated from DSS-treated $I l 18^{-/-}$mice also had impaired production of TNF $\alpha$, but not IL-1 $\beta$, indicating that TNF $\alpha$ production by Ly6 $\mathrm{C}^{\text {hi }}$ monocytes is, in part, IL-18 dependent (Figure 6c).

To determine whether inflammatory monocytes respond to IL-18 to promote TNF $\alpha$ production and resistance to DSS, Nlrp6 $6^{-1-}$ mice were also adoptively transferred with 
Mucosal immunology

a

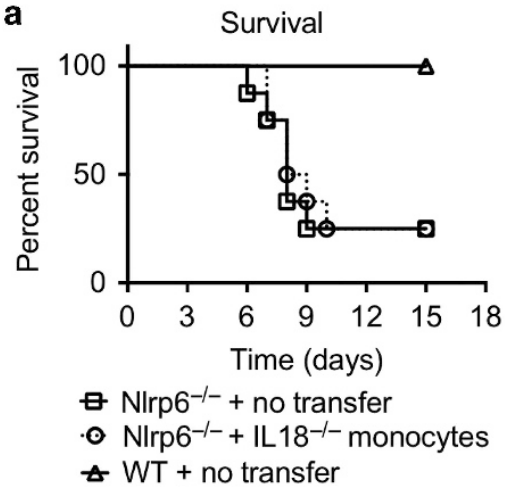

b

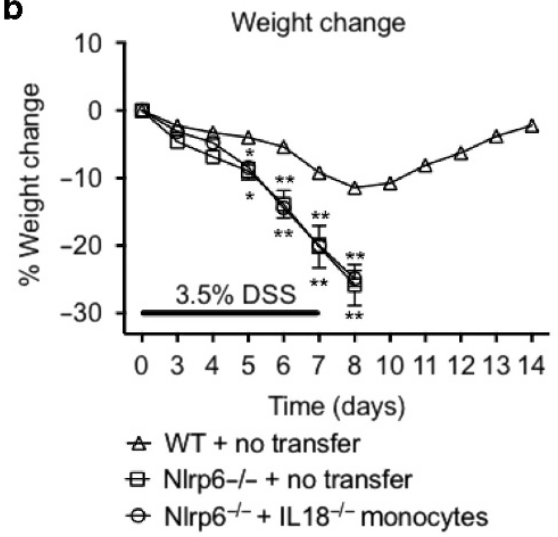

C

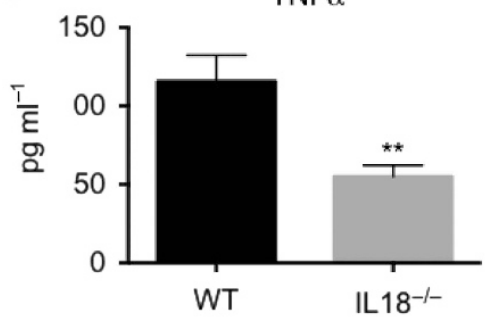

IL $1 \beta$

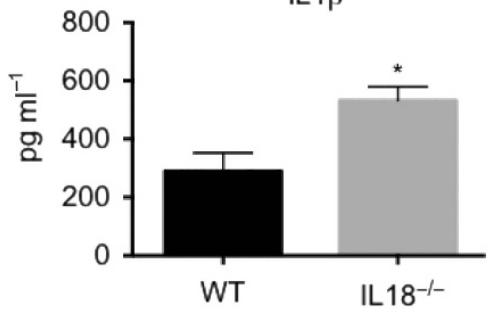

d

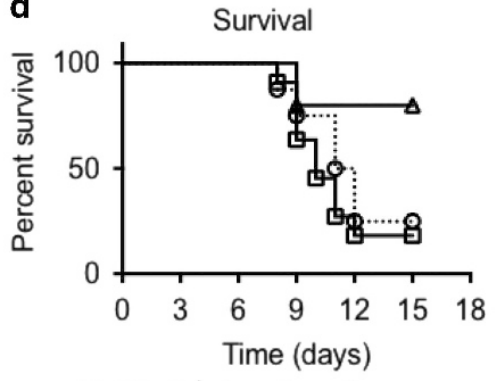

\# Nirp6 $6^{-1-}+$ no transfer

- $\mathrm{Nlrp6}^{-1-}+\mathrm{IL} 18 \mathrm{R}^{-1-}$ monocytes

$\star \mathrm{WT}+$ no transfer

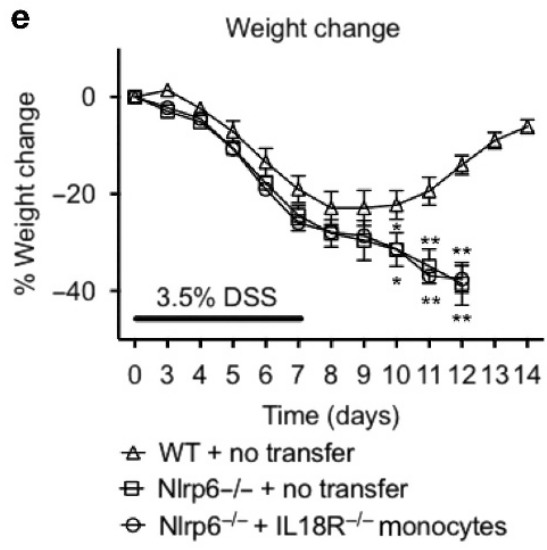

f

$\mathrm{TNF} \alpha$

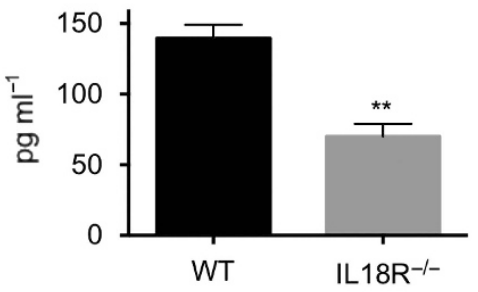

IL $1 \beta$

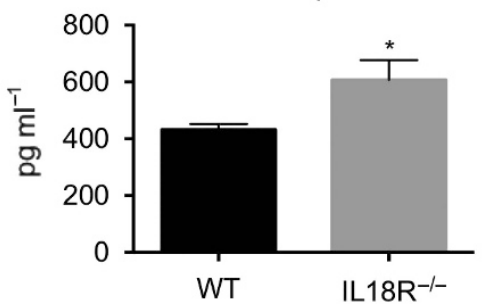

Figure 6 Autocrine IL-18 production by Ly6C $\mathrm{C}^{\text {hi }}$ monocytes protects against dextran sulfate sodium (DSS)-induced colitis by increasing TNF $\alpha$ production. Ly6C $\mathrm{C}^{\text {hi }}$ monocytes were purified from WT, $1118^{-/-}$or $I 118 \mathrm{r}^{-1 /}$ mice and i.v. injected $\left(2 \times 10^{6}\right.$ cells/per mouse $)$ into Nlrp6 ${ }^{-1-}$ recipients at day 3.5 of a 7-day course of 3.5\% DSS. Mice survival (a) and weight loss (b) for WT, NIrp6 ${ }^{-1-}$ and NIrp6 $6^{-1-}+1 / 18^{-1-}$ Ly6C $^{\text {hi }}$ monocytes was assessed. $n=8$ per group (c) Ly6C ${ }^{\text {hi }}$ monocytes were sorted from the lamina propria after 5 days of 2\% DSS (day 10 azoxymethane (AOM)/DSS model) from $1118^{-1-}$ mice, and cytokine levels in overnight cultures were measured by ELISA (enzyme-linked immunosorbent assay). $n=4$ per group. Mice survival (d) and weight loss (e) after adoptive transfer of $1 / 18 R^{-/-}$Ly6 $\mathrm{C}^{\text {hi }}$ monocytes into N/pr6 ${ }^{-/-}$mice. $n=5$ for WT, $n=11$ for N/rp6 ${ }^{-/-}$and $n=8$ for

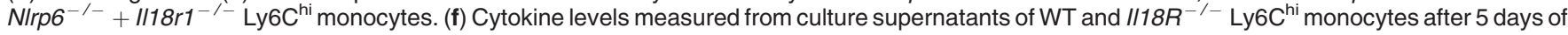
$2 \%$ DSS (day 10 AOM/DSS model), $n=4$ /group. ${ }^{\star} P<0.05$ and ${ }^{* \star} P<0.001$.

Il-18R ${ }^{-/-}$monocytes and survival measured after treatment with high-dose DSS. Nlrp6 $6^{-/-}$mice that received $\mathrm{Il}-18 \mathrm{R}^{-/-}$ monocytes were equally susceptible to DSS-induced mortality and weight loss as mock-treated Nlrp6 $6^{-/-}$mice (Figure 6d,e). Furthermore, cultures of Ly $6 \mathrm{C}^{\text {hi }}$ cells isolated from DSS-treated Illsr $1^{-/-}$mice also exhibited a defect in TNF $\alpha$ production, 
while secretion of IL-1 $\beta$ was actually increased rather than decreased (Figure 6f). These data strongly suggest that autocrine IL-18 signaling by inflammatory monocytes is important for regulating TNF $\alpha$ production early during the acute inflammatory response to DSS to promote resistance to intestinal injury and lethality.

\section{DISCUSSION}

NLRP6 is a member of the NLR family that is important for maintaining intestinal homeostasis. ${ }^{3}$ Our studies provide important insights into the mechanism by which NLRP6 protects against the development of colitis. We show that NLRP6 functions particularly in Ly6 $\mathrm{C}^{\text {hi }}$ inflammatory monocytes that are recruited to the intestinal LP during inflammation to protect the host against DSS-induced epithelial injury and subsequent life-threatening immunopathology. NLRP6 deficiency in inflammatory monocytes was also associated with defective TNF $\alpha$ production early in response to DSS, which, in turn, is important for reducing mortality. Our data further suggest that TNF $\alpha$ production is mediated by autocrine IL-18 signaling by Ly6 $\mathrm{C}^{\text {hi }}$ inflammatory monocytes. Therefore, we propose a model in which NLRP6 activation in inflammatory monocytes recruited in response to DSS-induced inflammation results in IL-18 secretion that upregulates their production of $\mathrm{TNF} \alpha$, which promotes epithelial repair and timely resolution of damage. ${ }^{15}$

Although IL-18 levels can be elevated in patients with active inflammatory disease, ${ }^{16,17}$ studies in mice have also demonstrated the protective effects of IL- 18 as $I 118^{-/-}$and Ill18r1 $1^{-/-}$ mice are more susceptible to colitis and colitis-associated colon tumorigenesis. ${ }^{18}$ This discrepancy may reflect differential roles of IL-18 that is dependent on timing and context. The production of IL-18 early during DSS-induced inflammation is important for epithelial restitution and resolution of inflammation, but in the setting of chronic, dysregulated inflammation, excessive IL-18 production can exacerbate disease. ${ }^{19}$ Consistently, impairment in IL-18 production during DSSinduced colitis by NLRP6-deficient mice is observed only early on during and after DSS treatment before the development of significant intestinal damage and inflammation. ${ }^{1}$ Our data demonstrates that Nlrp6 ${ }^{-1-}$ Ly6 $\mathrm{C}^{\text {hi }}$ monocytes in the LP also have impaired early IL-18 production. However, it is not currently known whether the reduced IL-18 secretion is due to decreased inflammasome-mediated caspase-1 activation or reflects a defect in a non-canonical mechanism that remains to be identified. In addition, the mechanism for IL-18-mediated protection is unclear. Previous studies suggested that IL-18 is predominantly expressed by the epithelium and that epithelialderived IL-18 is important for protection against DSS-induced damage. ${ }^{3,20}$ IL-18 production is also associated with upregulation of IL-22, which is implicated in epithelial repair. ${ }^{21}$ Our study suggests an additional mechanism for IL-18 function, which is to upregulate TNF $\alpha$ production in inflammatory monocytes.

TNF $\alpha$ levels can be significantly elevated in IBD patients, and TNF $\alpha$ antagonism is an approved therapy for IBD. The excessive production of $\mathrm{TNF} \alpha$ observed in patients with IBD and in mouse models of colitis likely reflects active disease that is already chronic and dysregulated. However, similar to IL-18, early production of $\mathrm{TNF} \alpha$ is important for tissue repair and maintaining intestinal homeostasis. ${ }^{15,22-24}$ Germ-free mice or mice that lack the adapter protein MyD88, which functions downstream of TLR-mediated bacterial sensing, have significant DSS-induced epithelial damage associated with the lack of induction of reparative cytokines, including $\mathrm{TNF} \alpha{ }^{15,25}$ In addition, TNF $\alpha \mathrm{Nn}$ addition, or mice that lack the adapter protein MyD88, which fun ${ }^{22,24} \mathrm{Tnfr} 1^{-1-}$ or Tnfr $2^{-/-}$mice as well as $\operatorname{Tnf\alpha ^{-/}}$ mice also develop more severe DSS-induced colitis with elevated levels of pro-inflammatory cytokines, increased epithelial damage, and increased mortality as compared with WT mice. ${ }^{24,26-28}$ Although there has been conflicting data that demonstrate a pathogenic role for TNFR2 in DSS-induced colitis, ${ }^{29}$ inflammatory monocytes are more likely to express TNFR1, while TNFR2 upregulation in colonic epithelial cells is observed in patients with inflammatory bowel disease. ${ }^{30,31}$ Altogether, these studies are consistent with our data since Nlrp6 $6^{-/-}$mice that receive rTNF $\alpha$ early during DSS treatment exhibit reduced intestinal inflammation and bacterial translocation.

We previously demonstrated that NLRP6 functions in a BMderived cell to limit inflammation-associated tumorigenesis. ${ }^{1}$ In the current study, we show that NLRP6 was induced specifically in myeloid cells within the LP during DSS-induced colitis. Furthermore, NLRP6 activity in Ly6 $\mathrm{C}^{\text {hi }}$ monocytes is important for reducing susceptibility to DSS-induced colitis. Despite the protection against the detrimental effects of inflammation, we did not see a statistically significant reduction in the number of tumors. Rather, the size of tumors was significantly decreased, suggesting that NLRP6 function in monocytes limits inflammation that primarily drives tumor growth. Consistently, the downregulation of inflammatory responses by removal of NF-kB activity in myeloid cells also resulted in reduced tumor size rather than tumor number. ${ }^{32}$ However, it is also possible that the administration of Ly6 $\mathrm{C}^{\text {hi }}$ monocytes only once with each DSS cycle is insufficient to affect tumor number, or that another BM-derived cell in addition to monocytes is necessary for protection.

There are two major populations of monocytes in the murine intestinal LP, classical Ly6C ${ }^{\text {hi }}$ inflammatory monocytes and Ly6C ${ }^{-}$monocytes that differentiate into macrophages. ${ }^{11}$ Ly6C ${ }^{\text {hi }}$ monocytes can be further differentiated by their expression level of CX3CR1. ${ }^{33,34}$ LP Ly6C ${ }^{\text {hi }}$ monocytes can differentiate into $\mathrm{CX} 3 \mathrm{CR}^{\text {hi }}$ resident macrophages or effector CX3CR $1^{\text {int }}$ inflammatory monocytes whose primary function is to rapidly produce pro-inflammatory cytokines after their recruitment to sites of inflammation and injury. Our data strongly indicates that during DSS-induced inflammation, the majority of Ly6 $\mathrm{C}^{\text {hi }}$ monocytes that are recruited to the LP express CX3CR1 and adoptively transferred Ly6C ${ }^{\text {hi }}$ inflammatory monocytes recruited to the intestinal LP are predominantly Ly6 $\mathrm{C}^{\text {hi }} \mathrm{CX} 3 \mathrm{CR} 1^{\text {int }}$ (Supplementary Figure 3 ), consistent with previous studies. ${ }^{35}$ Although inflammatory monocytes have been implicated in exacerbating colitis since 
they are capable of producing pro-inflammatory factors, and are found in increased numbers in persistently inflamed tissue, ${ }^{11,36}$ they also have important roles in restoring homeostasis and limiting damage. This is largely through their ability to be early responders to tissue injury and produce cytokines, including TNF $\alpha$ and IL18 that have reparative functions thereby allowing epithelial restitution and resolution of inflammation. ${ }^{9}$ Consistently, depletion of intestinal monocytes in WT mice resulted in increased susceptibility to colitis. ${ }^{37}$ Our data further highlight a positive impact by Ly6C $\mathrm{C}^{\mathrm{hi}}$ monocytes via NLRP6 as an early source of TNF $\alpha$ to prevent uncontrolled inflammation in response to epithelial damage. NLRP6 deficiency in Ly6 $\mathrm{C}^{\text {hi }}$ monocytes is also associated with defective ROS production, which may further limit translocation of bacteria and exacerbation of intestinal inflammation. ${ }^{38}$ Indeed, the adoptive transfer of Ly6 $\mathrm{C}^{\text {hi }}$ monocytes resulted in reduced bacterial translocation and improved barrier function. It is possible that the decreased ROS production is related to defective production of TNF $\alpha$, which is needed to promote the assembly of the NADPH oxidase complex that produces ROS. ${ }^{39}$ Nonetheless, these results are somewhat surprising in light of the study by Anand et al., which suggests that NLRP6 negatively regulates cytokine responses, including TNF $\alpha$, in BM-derived macrophages (BMDMs) after exposure to bacterial ligands. ${ }^{12}$ One possible explanation for this disparate finding is that BMDMs cultured in vitro represent a population distinct from inflammatory monocytes. In fact, we demonstrate that not all monocyte populations regulate TNF $\alpha$ production via NLRP6 as we observed no difference in TNF $\alpha$ production between WT and $N l r p 6^{-1-}$ LP resident macrophages. Thus, it is likely that differential TNF $\alpha$ responses reflect not only differences in the particular cell population and location, but also the context in which inflammation occurs.

How NLRP6 promotes TNF $\alpha$ production in inflammatory monocytes remains to be fully elucidated. The lack of a known NLRP6 receptor agonist has made the identification of cognate signaling pathways challenging. However, our data suggest that the mechanism may be mediated indirectly through the regulation of IL-18 production in monocytes. The adoptive transfer of $\mathrm{Ill}^{-/-}$or $\mathrm{Il18r1^{-/- }}$ monocytes into Nlrp6 $6^{-/-}$ mice failed to rescue DSS-induced mortality, suggesting that autocrine IL-18 signaling by inflammatory monocytes is important for protection against DSS-induced colitis. Furthermore, both $I l 18^{-/-}$and Il18r1 ${ }^{-/-}$monocytes isolated from DSS-treated mice have impaired production of TNF $\alpha$ similar to that observed with $N l r p 6^{-1-}$ monocytes, consistent with a role for NLPR6 in regulating TNF $\alpha$ production in monocytes via IL-18. Although IL-18 is typically associated with the upregulation of Th1 responses, and in particular, IFN $\gamma^{40}$ IL-18 has also been shown to activate monocytes to enhance TNF $\alpha$ production, ${ }^{41,42}$ consistent with our data.

In summary, our studies highlight a previously unrecognized role for NLRP6 in modulating IL-18-dependent TNF $\alpha$ production in inflammatory Ly6 $\mathrm{C}^{\text {hi }}$ monocytes that can act as early responders to an inflammatory insult, thereby limiting bacterial translocation, promoting timely epithelial repair, and preventing the onset of dysregulated inflammation. Our data additionally suggest that early production of IL- 18 to promote resistance against DSS-induced colitis is mediated not only by the intestinal epithelium, but also by inflammatory monocytes through NLRP6. Together, these results suggest that modulation of NLRP6 activity may be a potential strategy for the prevention and treatment of inflammatory bowel disease.

\section{METHODS}

Animal procedures. Adult 7-11 weeks old C57BL/6 WT, Nlrp $6^{-/-}$, $\mathrm{Il18}^{-/-}$or Il18r1 ${ }^{-/-}$, and $\mathrm{GFP}^{+}$transgenic C57BL/6 (C57BL/6$\mathrm{Tg}(\mathrm{UBC}-\mathrm{GFP}) 30 \mathrm{Sch} / \mathrm{J})$ mice were bred in-house under specific pathogen-free conditions at the University of Michigan. WT, $I l 18^{-/-}$ and Ill18r1 $1^{-1-}, \mathrm{GFP}^{+}, \mathrm{CCR} 2^{-/-}$, and CX3CR1 $1^{\text {gfp }}$ mice (all B6 background) were originally purchased from Jackson (Bar Harbor, ME). Nlrp $6^{-1-}$ in B6 background were previously described. ${ }^{1}$ Animal studies were conducted under protocols approved by the University of Michigan Committee on the Use and Care of Animals.

DSS-induced colitis and AOM/DSS-induced tumorigenesis. To induce colitis and lethality, mice were treated with 3.5\% DSS (MP Biomedicals; m.w. 36,000-50,000Da) in regular drinking water for 7 days. To develop colitis-associated tumors, mice were first injected with $10 \mathrm{mg} \mathrm{kg}^{-1} \mathrm{AOM}$ (Sigma) i.p., followed by 5 days of $2 \%$ DSS starting on day 5. Mice were allowed to recover for 16 days with regular drinking water, and then treated with two additional cycles of $2 \%$ DSS (Figure 2a). Mice were sacrificed after the last cycle of DSS for tumor counting and size measurement by calipers.

Isolation of lamina propria cells. LP cells were isolated as previously described. ${ }^{1}$ Briefly, colons were cut into small pieces in HBSS buffer (Gibco) supplemented with 2.5\% heat-inactivated FBS (Gibco, Gran Island, NY) (HBSS + ), and washed with magnetic stirring at $37^{\circ} \mathrm{C}$. Colon pieces were then incubated in HBSS $+/ 1 \mathrm{mM}$ DTT at $37^{\circ} \mathrm{C}$ followed by additional washes and incubation in $\mathrm{HBSS}+/ 1 \mathrm{mM}$ EDTA. The supernatant containing IECs and IELs was saved for further analysis. The remaining colon pieces were further digested with $400 \mathrm{IU} \mathrm{ml}^{-1}$ Type III collagenase (Worthington) and $10 \mathrm{~g} \mathrm{ml}^{-1}$ DNase I (Worthington) for two hours at $37^{\circ} \mathrm{C}$. When all tissue was digested, the cell suspension was filtered through a $70 \mu \mathrm{m}$ filter, before running on a $75 \% / 40 \%$ Percoll gradient to collect enriched LP cells from the interface.

Flow cytometry. Cell suspensions were treated with anti-CD16/CD32 $(2.4 \mathrm{G} 2)$ and then surface stained with combinations of the following fluorochrome-conjugated antibodies: from Biolegend (San Diego, CA): CD3e-FITC (clone: 145-2C11), Ly6C-FITC or APC/Cy7 (clone: HK1.4), Ly6G-PE/Cy7 (clone: 1A8), CD3e-APC/Cy7 (clone: 1452C11), CD45R/B220-Pacific Blue (clone: RA3-6B2), CCR2-AlexaFluor647 (clone: SA203G11), CX3CR1-APC (clone: SA011F11), IgG2A,K-APC isotype control; from BD Biosciences (San Jose, CA): NK1.1-PerCP/Cy5.5 (clone: PK136); from Ebiosciences (San Diego, CA): F4/80-APC (clone: BM8), CD4 (L3T4)-FITC (clone: RM4-5), CD11b-FITC (clone: M1/70), CD11b-PE (clone: M1/70), CD4-APC (clone: GK1.5). Cells were acquired on FACSCanto or AriaIII flow cytometers using FACSDiVa software, and data were analyzed using FlowJo software (Tree Star, Ashland, OR).

Adoptive transfer experiments. WT, $C \mathrm{cr} 2^{-1-}$ or CX3CR1 $1^{\mathrm{gfp}}$ mice were used as donors. Single-cell suspensions were prepared from flushed BM in fully supplemented RPMI followed by lysis of red blood cells and isolation of Ly6C $\mathrm{C}^{\text {hi }}$ monocytes $\left(\mathrm{CD} 3^{-} \mathrm{CD} 11 \mathrm{~b}^{+}\right.$ Ly6 $\mathrm{C}^{\text {hi }} \mathrm{Ly}_{6 \mathrm{G}^{-}}^{-}$) by FACs sorting. Two million cells were injected i.v. into $\mathrm{Nlrp6}^{-1-}$, WT or $\mathrm{Ccr} 2^{-9-}$ recipient mice.

Treatment of mice with recombinant TNF $\alpha$. WT or Nlrp6 $6^{-/-}$mice were administered $1 \mu \mathrm{g}$ of sterilely filtered recombinant mouse 
TNF $\alpha$ (PreproTech) i.p. on days 3-7 in the 3.5\% DSS colitis model (days $3-7$ ) or days $8-10$ in the AOM/DSS colitis and tumorigenesis model.

Assessment of colon inflammation. Colons were flushed free of feces, opened longitudinally, and jelly rolled for formalin fixation and paraffin embedding. Five- $\mu \mathrm{m}$ sections were used for $\mathrm{H} \& \mathrm{E}$ staining. Histological assessment was performed in a blinded fashion by a pathologist (KE) using a previously described scoring system ${ }^{1}$ with modifications. Entire colon sections were assessed for three main parameters: inflammatory cell infiltration, hyperplasia, and epithelial damage that were individually scored (0 to 4), (Supplementary Table 1). For each mouse, a weighted percent average based on the number of fields with a certain score was calculated for each parameter and then summed to obtain the final score. Assessment of colon weight after DSS treatment was performed by measuring the colon weight (excluding the cecum) after removal of feces normalized by its length $(\mathrm{cm})$.

Intestinal permeability. Mice were fasted for $8 \mathrm{~h}$ with the exception of drinking water prior to the administration of $0.6 \mathrm{mg} \mathrm{kg}^{-1}$ FITC-dextran (4kDa; Sigma-Aldrich, St. Louis, MO) by oral gavage. Serum was collected $4 \mathrm{~h}$ later, diluted 1:3 in phosphatebuffered saline (PBS), and fluorescence measured using a fluorescent spectrophotometer with emission at $488 \mathrm{~nm}$ and absorption at $525 \mathrm{~nm}$.

Reverse transcription and qPCR. Total RNA was isolated from LP, BM cells, or from sorted populations of LP cells using the Nucleospin RNA kit (Machery-Nagel, Bethlehem, PA). cDNA synthesis was performed using iScript (Bio-Rad, Hercules, CA), and cDNA was used for quantitative PCR (qPCR) using the SYBR Green Master Mix (Applied Biosystems, Foster City, CA) on the ABI 7900HT (Applied Biosystems). Ct values were normalized to the $\beta$-actin gene. Primer sequences are available upon request.

ELISA. To measure fecal lipocalin-2 levels, fecal samples were homogenized in PBS at $100 \mathrm{mg} \mathrm{ml}^{-1}$ and supernatant was further diluted in a range of 1:500 to 1:5,000 for lipocalin-2/NGAL enzyme-linked immunosorbent assay (ELISA; R\&D Systems, Minneapolis, MN). TNF $\alpha$, IL-6 and IL- $1 \beta$ ELISAs (all R\&D Systems) were performed with 24-hour culture supernatants of Ly6C ${ }^{\text {hi }}$ monocytes.

Measurement of ROS. Total cellular ROS were measured by CellROX Deep Red reagent kit (Invitrogen) according to the manufacturer's protocol. Briefly, upon surface staining for flow cytometry, cells were resuspended in $200 \mu \mathrm{l}$ of pre-warmed $\mathrm{HBSS}+2.5 \mathrm{mM}$ Probenecid + CellROX, incubated for 30 minutes at $37^{\circ} \mathrm{C}$ (in the dark), washed and acquired on FACSCanto or AriaIII flow cytometers (BD Bioscience). CellROX Deep Red has peak excitation and emission at 640 and $665 \mathrm{~nm}$.

Phagocytosis assay. eGFP-expressing E. coli (K12) was a kind gift from Gabriel Nunez (University of Michigan). Ly6C ${ }^{\text {hi }}$ monocytes were specifically sorted from WT or Nlrp6 $6^{-/-}$mice at day 10 of $\mathrm{AOM} / \mathrm{DSS}$ and incubated for $1.5 \mathrm{~h}$ with $E$. coli-GFP at multiplicity of infection (MOI) $0,5,25$, or 60. Zombie Violet viability die (Biolegend) was used to identify live Ly6C $\mathrm{C}^{\text {hi }}$ monocytes, which phagocytized $\mathrm{GFP}^{+}$E. coli using FACSCanto or AriaIII flow cytometers (BD Bioscience).

Bacterial translocation. MLNs were homogenized in PBS by passing through a $70 \mu \mathrm{m}$ strainer and then plated on Trypticase Soy Agar with $5 \%$ Sheep Blood (TSA II) plates (BD) to determine CFUs after 24-h incubation under aerobic conditions or 48-h under anaerobic conditions. Total DNA was extracted using the Nucleospin kit (MacheryNagel), and bacterial load quantified by qPCR the universal 16S rRNA gene primers EUB-For- $5^{\prime}$-AGAGTTTGATCCTGGCTC- $3^{\prime}$ and EUBRev-5'-TGCTGCCTCCCGTAGGAGT-3'.
Statistical analysis. Statistically significant differences were determined using two-way analysis of variance (ANOVA) with a Bonferroni post hoc test (time $\times$ genotype, $P<0.05$ ), by one-way ANOVA with a Student-Newman-Keuls post hoc test $(P$ value $<0.05)$, or by twotailed Student's unpaired $t$-test when only two groups are compared. Survival curve comparison was performed by Mantel-Cox log-rank test. Kruskal-Wallis one-way analysis of variance non-parametric test was used for non-continuous variables. Data are shown as mean \pm s.e.m. GraphPad Prism6 software was utilized for statistical analysis. $^{43-45}$

SUPPLEMENTARY MATERIAL is linked to the online version of the paper at http://www.nature.com/mi

\section{ACKNOWLEDGMENTS}

This work was supported by the National Institutes of Health grant R01CA166879 and American Cancer Society Research Scholar Grant awarded to GYC, National Institutes of Health grant F32CA200144 awarded to SSS and by grant number UL1TR000433 from the National Center for Advancing Translational Sciences awarded to SSS.

\section{DISCLOSURE}

The authors declared no conflict of interest.

(c) 2017 Society for Mucosal Immunology

\section{REFERENCES}

1. Chen, G.Y., Liu, M., Wang, F., Bertin, J. \& Nunez, G. A functional role for Nlrp6 in intestinal inflammation and tumorigenesis. J. Immunol. 186, 7187-7194 (2011).

2. Chen, G.Y. Role of Nlrp6 and Nirp12 in the maintenance of intestinal homeostasis. Eur. J. Immunol. 44, 321-327 (2013).

3. Elinav, E. et al. NLRP6 inflammasome regulates colonic microbial ecology and risk for colitis. Cell 145, 745-757 (2011).

4. Normand, S. et al. Nod-like receptor pyrin domain-containing protein 6 (NLRP6) controls epithelial self-renewal and colorectal carcinogenesis upon injury. Proc. Natl. Acad. Sci. USA 108, 9601-9606 (2011).

5. Allen, I.C. et al. The NLRP3 inflammasome functions as a negative regulator of tumorigenesis during colitis-associated cancer. J. Exp. Med. 207, 1045-1056 (2010).

6. Zaki, M.H., Boyd, K.L., Vogel, P., Kastan, M.B., Lamkanfi, M. \& Kanneganti, T.D. The NLRP3 inflammasome protects against loss of epithelial integrity and mortality during experimental colitis. Immunity 32, 379-391 (2010).

7. Wlodarska, M. et al. NLRP6 inflammasome orchestrates the colonic hostmicrobial interface by regulating goblet cell mucus secretion. Cell 156, 1045-1059 (2014).

8. Gordon, S. \& Taylor, P.R. Monocyte and macrophage heterogeneity. Nat. Rev. Immunol. 5, 953-964 (2005).

9. Zigmond, E. et al. Ly6C hi monocytes in the inflamed colon give rise to proinflammatory effector cells and migratory antigen-presenting cells. Immunity 37, 1076-1090 (2012).

10. Sherwood, R.A. Faecal markers of gastrointestinal inflammation. J. Clin. Pathol. 65, 981-985 (2012).

11. Murray, P.J. \& Wynn, T.A. Protective and pathogenic functions of macrophage subsets. Nat. Rev. Immunol. 11, 723-737 (2011).

12. Anand, P.K. et al. NLRP6 negatively regulates innate immunity and host defence against bacterial pathogens. Nature 488, 389-393 (2012).

13. Kempster, S.L. et al. Developmental control of the Nirp6 inflammasome and a substrate, IL-18, in mammalian intestine. Am. J. Physiol. Gastrointest. Liver Physiol. 300, G253-G263 (2010).

14. Reinecker, H.C. et al. Enhanced secretion of tumour necrosis factor-alpha, $\mathrm{IL}-6$, and $\mathrm{L}-1$ beta by isolated lamina propria mononuclear cells from patients with ulcerative colitis and Crohn's disease. Clin. Exp. Immunol. 94, 174-181 (1993)

15. Rakoff-Nahoum, S., Paglino, J., Eslami-Varzaneh, F., Edberg, S. \& Medzhitov, R. Recognition of commensal microflora by toll-like receptors is required for intestinal homeostasis. Cell 118, 229-241 (2004). 
16. Pizarro, T.T. etal. IL-18, a novel immunoregulatory cytokine, is up-regulated in Crohn's disease: expression and localization in intestinal mucosal cells. J. Immunol. 162, 6829-6835 (1999).

17. Furuya, D. et al. Serum interleukin-18 concentrations in patients with inflammatory bowel disease. J. Immunother. 25, S65-S67 (2002).

18. Salcedo, R. et al. MyD88-mediated signaling prevents development of adenocarcinomas of the colon: role of interleukin 18. J. Exp. Med. 207, 1625-1636 (2010).

19. Siegmund, B. Interleukin-18 in intestinal inflammation: friend and foe? Immunity 32, 300-302 (2010).

20. Dupaul-Chicoine, J. et al. Control of intestinal homeostasis, colitis, and colitis-associated colorectal cancer by the inflammatory caspases. Immunity 32, 367-378 (2010).

21. Huber, S. et al. IL-22BP is regulated by the inflammasome and modulates tumorigenesis in the intestine. Nature 491, 259-263 (2012).

22. Naito, Y. et al. Enhanced intestinal inflammation induced by dextran sulfate sodium in tumor necrosis factor-alpha deficient mice. J. Gastroenterol. Hepatol. 18, 560-569 (2003).

23. Noti, M., Corazza, N., Mueller, C., Berger, B. \& Brunner, T. TNF suppresses acute intestinal inflammation by inducing local glucocorticoid synthesis. J. Exp. Med. 207, 1057-1066 (2010).

24. Wang, Y. et al. Protective role of tumor necrosis factor (TNF) receptors in chronic intestinal inflammation: TNFR1 ablation boosts systemic inflammatory response. Lab. Invest. 93, 1024-1035 (2013).

25. Zhan, Y. et al. Gut microbiota protects against gastrointestinal tumorigenesis caused by epithelial injury. Cancer Res. 73, 7199-7210 (2013).

26. Edelblum, K.L., Goettel, J.A., Koyama, T., McElroy, S.J., Yan, F. \& Polk, D.B. TNFR1 promotes tumor necrosis factor-mediated mouse colon epithelial cell survival through RAF activation of NF-kappaB. J. Biol. Chem. 283, 29485-29494 (2008).

27. Feng, Y. \& Teitelbaum, D.H. Tumour necrosis factor-induced loss of intestinal barrier function requires TNFR1 and TNFR2 signalling in a mouse model of total parenteral nutrition. J. Physiol. 591, 3709-3723 (2013).

28. Stillie, R. \& Stadnyk, A.W. Role of TNF receptors, TNFR1 and TNFR2, in dextran sodium sulfate-induced colitis. Inflamm. Bowel Dis. 15, 1515-1525 (2009).

29. Wang, K. et al. Opposite role of tumor necrosis factor receptors in dextran sulfate sodium-induced colitis in mice. PLoS One 7, e52924 (2012).

30. Naude, P.J., den Boer, J.A., Luiten, P.G. \& Eisel, U.L. Tumor necrosis factor receptor cross-talk. FEBS J. 278, 888-898 (2011).
31. Hijdra, D., Vorselaars, A.D., Grutters, J.C., Claessen, A.M. \& Rijkers, G.T. Differential expression of TNFR1 (CD120a) and TNFR2 (CD120b) on subpopulations of human monocytes. J. Inflamm. (Lond) 9, 38 (2012).

32. Greten, F.R. et al. IKKKbeta links inflammation and tumorigenesis in a mouse model of colitis-associated cancer. Cell 118, 285-296 (2004).

33. Bain, C.C. \& Mowat, A.M. The monocyte-macrophage axis in the intestine. Cell Immunol. 291, 41-48 (2014).

34. Bain, C.C. \& Mowat, A.M. Macrophages in intestinal homeostasis and inflammation. Immunol. Rev. 260, 102-117 (2014).

35. Bain, C.C. et al. Resident and pro-inflammatory macrophages in the colon represent alternative context-dependent fates of the same Ly6Chi monocyte precursors. Mucosal Immunol. 6, 498-510 (2013).

36. Varol, C., Zigmond, E. \& Jung, S. Securing the immune tightrope: mononuclear phagocytes in the intestinal lamina propria. Nat. Rev. Immunol. 10, 415-426 (2010).

37. Qualls, J.E., Kaplan, A.M., van Rooijen, N. \& Cohen, D.A. Suppression of experimental colitis by intestinal mononuclear phagocytes. J. Leukoc. Biol. 80, 802-815 (2006)

38. Molloy, M.J. et al. Intraluminal containment of commensal outgrowth in the gut during infection-induced dysbiosis. Cell Host Microbe 14, 318-328 (2013).

39. Bayir, H. Reactive oxygen species. Crit. Care Med. 33, S498-S501 (2005).

40. Nakanishi, K., Yoshimoto, T., Tsutsui, H. \& Okamura, H. Interleukin-18 regulates both Th1 and Th2 responses. Annu. Rev. Immunol. 19, 423-474 (2001).

41. Dai, S.M., Matsuno, H., Nakamura, H., Nishioka, K. \& Yudoh, K. Interleukin18 enhances monocyte tumor necrosis factor alpha and interleukin-1beta production induced by direct contact with T lymphocytes: implications in rheumatoid arthritis. Arthritis Rheum. 50, 432-443 (2004).

42. Dias-Melicio, L.A., Fernandes, R.K., Rodrigues, D.R., Golim, M.A. \& Soares, A.M. Interleukin-18 increases TLR4 and mannose receptor expression and modulates cytokine production in human monocytes. Mediators Inflamm. 2015, 236839 (2015).

43. Ahmadian, M. et al. PPARgamma signaling and metabolism: the good, the bad and the future. Nat. Med. 19, 557-566 (2013).

44. Adachi, M. et al. Peroxisome proliferator activated receptor gamma in colonic epithelial cells protects against experimental inflammatory bowel disease. Gut 55, 1104-1113 (2006).

45. Shah, Y.M., Morimura, K. \& Gonzalez, F.J. Expression of peroxisome proliferator-activated receptor-gamma in macrophage suppresses experimentally induced colitis. Am. J. Physiol. Gastrointest. Liver Physiol. 292, G657-G666 (2007). 